\title{
Riñón único funcionante, ¿riesgo de enfermedad renal crónica?
}

\author{
Single functioning kidney, risk of chronic kidney disease?
}

La condición de riñón único o solitario puede ser congénita o adquirida, real o funcional. Dentro de las congénitas se encuentran la agenesia renal, la displasia renal multiquística unilateral (DRMU) y la hidronefrosis congénita, por mencionar las más frecuentes, y dentro de las formas adquiridas, la exclusión renal y nefrectomía por diversas causas y el riñón trasplantado.

Muchos estudios, se han realizado sobre el seguimiento de los pacientes receptores de trasplante renal de donantes vivo o cadavérico, que muestran deterioro de la tasa de filtración glomerular (TFG) a largo plazo y proteinuria persistente (1); sin embargo, debido a que las personas tuvieron una enfermedad crónica durante varios años y reciben medicamentos inmunosupresores, no son comparables con otros casos de riñón único funcionante.

En este número de la revista se publica el artículo de investigación de Villarreal A. et al. (2), cuyo objetivo fue determinar la tasa de filtración glomerular y los niveles de proteinuria en 24 horas en pacientes con un solo riñón, con un tiempo mínimo de seguimiento de 5 años. Se incluyeron 50 niños entre 5 y 13,5 años; 25 correspondieron a displasia renal multiquística unilateral (DRMU), 16 a agenesia renal y 9 que habían sido sometidos a nefrectomía. Se encontró que la mitad presentó proteinuria significativa persistente y ninguno proteinuria masiva, y no hubo diferencia entre las etiologías. El 58\% tenía disminución de la tasa de filtración glomerular, pero las 3/4 partes, en estadio I.

La introducción y el desarrollo de la nefrectomía laparoscópica, aunado con el aumento de la demanda de órganos, ha generado un incremento de los trasplantes con donante vivo a nivel mundial. Como consecuencia de ello, un aumento considerable de personas sanas con un solo riñón, lo que ha generado gran interés por conocer cuál es el riesgo de morbilidad y mortalidad a largo plazo (3).

Textor SC, et al. (4), encontraron que a corto plazo (un año de donación), los sujetos con hipertensión esencial moderada y función renal normal, no tuvieron efectos sobre la presión arterial, TFG y la excreción urinaria de proteínas. Por otro lado, Ibrahim HN, et al. (5), encontraron que a largo plazo, la sobrevida renal de los donantes fue similar al de los controles apareados por edad, sexo y raza o grupo étnico. En un tiempo de seguimiento promedio de $12,2 \pm 9,2$ años después de la donación, el $85,5 \%$ tenía TFG $\geq 60 \mathrm{ml} / \mathrm{min} \times 1,73 \mathrm{~m}^{2} \mathrm{SC} ; 32,1 \%$ hipertensión, y $12,7 \%$ albuminuria.

Las causas de mortalidad a largo plazo en donantes de riñón son los mismos que los observados en la población general: cardiovasculares, cáncer y accidentes de tránsito, y la tasa de mortalidad es menor que la esperada para la población general cuando se ajusta a edad y sexo $(6,7)$. Por lo tanto, en individuos sanos con función renal adecuada y sin factores de riesgo adicionales como obesidad, diabetes, hipertensión arterial, etc., quedar con un solo riñón funcionante, no los pone en riesgo de nefropatía a largo plazo (3).

Sin embargo, como hay estudios que muestran que algunos donantes de riñón pueden presentar un leve incremento de la presión arterial y de la excreción de proteínas en orina, se recomienda control periódico clínico regular, en las personas con riñón único funcionante, que incluya medición de la tasa de la TFG y de la proteinuria en 24 horas. Es importante recordar que las fórmulas para estimar la TFG a partir de la creatinina, se han desarrollado en base a personas con ambos riñones. 


\section{REFERENCIAS BIBLIOGRÁFICAS}

1. Jiménez del Cerro LA, Franco A, Rivera F, Olivares J. Evolución de la función renal en el trasplante renal. Factores predictivos del deterioro funcional. NEFROLOGÍA. 2001; XXI(3):295-304.

2. Villarreal AE, Ferrándiz RA, Salinas CE, Loza CA, García JJ, Higueras WA. Evolución de la tasa de filtración glomerular y proteinuria a largo plazo en niños monorrenos en un hospital nacional. Rev Med Hered. 2020; 31:5-11.

3. Oppenheimer F. Short, medium, and longterm follow-up of living donors. Nefrología 2010; 30(Suppl.2):100-5. doi:10.3265/Nefrologia.
pre2010.Nov.10699

4. Textor SC, Taler SJ, Driscoll N, Larson TS, Gloor $\mathrm{JM}$, Griff in $\mathrm{M}$, et al. Blood pressure and renal function after kidney donation from hypertensive living donors. Transplantation. 2004; 78(2):276-82.

5. Ibrahim HN, Foley R, Tan L, Rogers T, Bailey $\mathrm{RF}$, Guo $\mathrm{H}$, et al. Long-term consequences of kidney donation. N Engl J Med. 2009; 360(5):45969.

6. Davis CL. Living kidney donors: current state of affairs. Adv Chronic Kidney Dis. 2009; 16(4):242-9.

7. Felipe C, Oppenheimer F, Plaza JJ. Trasplante renal de vivo: una opción terapéutica real. Nefrologia. 2000; 20(1):8-21. 